\title{
Populations of OB-type stars in galaxies
}

\section{Christopher J. Evans}

\author{
UK Astronomy Technology Centre, Blackford Hill, Edinburgh, EH9 3HJ, UK \\ email: chris.evans@stfc.ac.uk
}

\begin{abstract}
One of the challenges for stellar astrophysics is to reach the point at which we can undertake reliable spectral synthesis of unresolved populations in young, star-forming galaxies at high redshift. Here I summarise recent studies of massive stars in the Galaxy and Magellanic Clouds, which span a range of metallicities commensurate with those in high-redshift systems, thus providing an excellent laboratory in which to study the role of environment on stellar evolution. I also give an overview of observations of luminous supergiants in external galaxies out to a remarkable $6.7 \mathrm{Mpc}$, in which we can exploit our understanding of stellar evolution to study the chemistry and dynamics of the host systems.
\end{abstract}

Keywords. Galaxy: stellar content, Magellanic Clouds, stars: early-type, stars: fundamental parameters

\section{Introduction}

One of the prime motivations to study stellar evolution is to use that knowledge to develop tools to explain integrated-light observations of distant star clusters and galaxies. Consider the recent multi-wavelength study of a gravitationally-lensed galaxy at a redshift of $z=2.3$ by Swinbank et al. (2010). By virtue of the lens, individual regions are resolved in sub-millimetre imaging, each $\sim 100 \mathrm{pc}$ in scale. These are intense regions of star formation on a comparable spatial scale to that of 30 Doradus, viewed at a time when the universe was significantly younger. Such observations are only possible at present due to the magnification of the lens but, with future facilities such as the Atacama Large Millimetre Array (ALMA) and Extremely Large Telescopes (ELTs), we can expect comparable observations in unlensed systems in the coming years. One of the real tests of stellar astrophysics is to reach the point at which we are confident that we can interpret integrated-light spectroscopy of such distant systems accurately, exploiting our understanding of massive stars to obtain new insights into the processes at work during one of the most critical epochs of galaxy evolution.

Population synthesis codes such as Starburst99 (Leitherer et al. 1999) are the 'bridge' from studies of individual stars to analysis of entire populations on galaxy scales. The rest-frame ultraviolet (UV) is rich with the signatures of stellar winds and, for highredshift galaxies, is redshifted into the optical (e.g. Pettini et al. 2002) and, ultimately, into the near-infrared for the most distant systems. To model the rest-frame UV a new spectral library for Starburst99 has been calculated by Leitherer et al. (2010) using the WM-Basic model atmosphere code (Pauldrach, Hoffmann \& Lennon, 2001). Fig. 1 shows a comparison of library spectra from the International Ultraviolet Explorer (IUE) with spectra calculated using the new WM-Basic library. In general there is excellent agreement, a worthy testament to theoretical developments in recent years! Many of the apparent differences are related to observational issues such as narrow interstellar features and the significant wings of the Lyman- $\alpha$ absorption from Galactic H I. The most discrepant stellar feature is O v $\lambda 1371 \AA$, known to be sensitive to wind inhomogeneities ('clumping'), the effects of which are not incorporated in WM-Basic at present. 


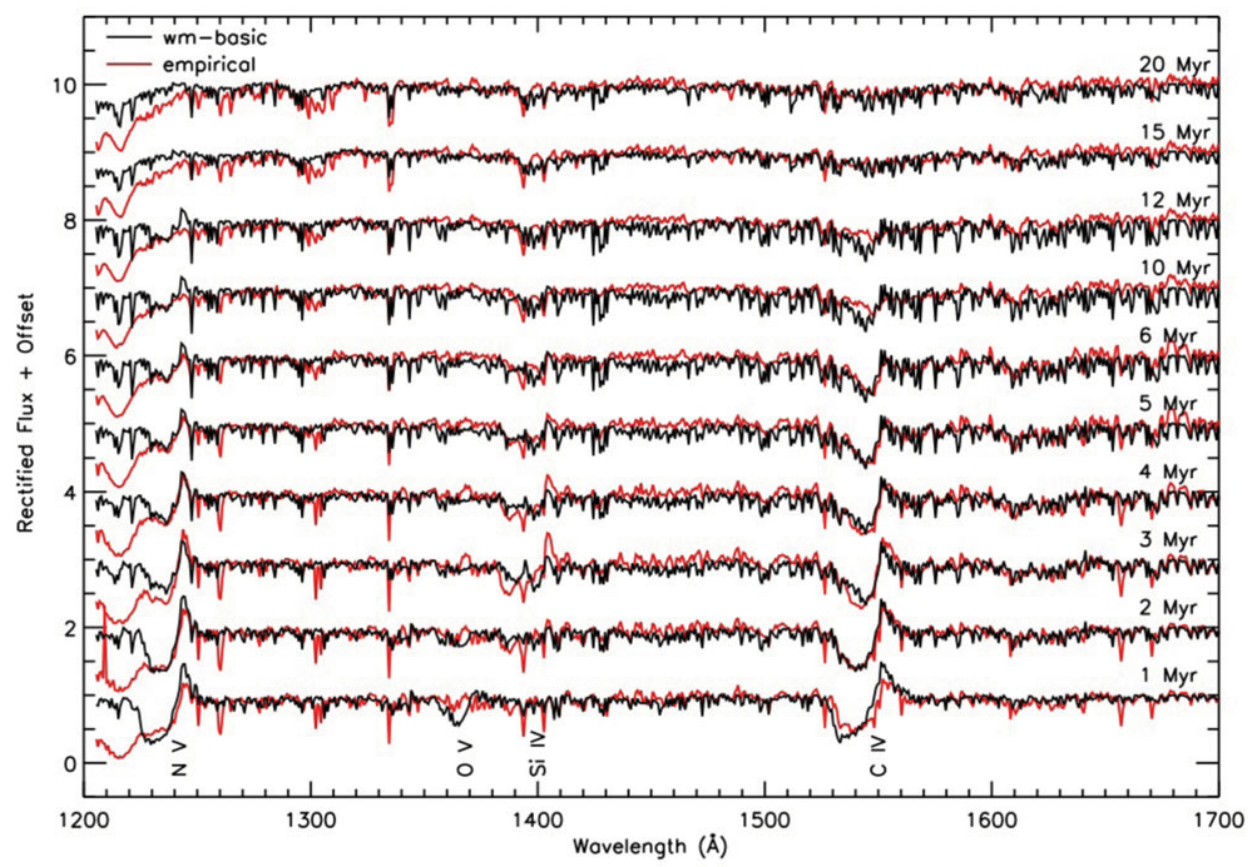

Figure 1. Single-stellar populations from the new Starburst99 WM-Basic library (solar metallicity, black lines) compared to empirical IUE templates (red lines), from Leitherer et al. (2010).

The comparison in Fig. 1 reminds me of comment that a high-redshift astronomer once said to me at a conference: '... but stars are done aren't they?' At the time I argued strongly to the contrary - while we now have a significant command of stellar astrophysics, a broad range of fundamental questions still eludes us for massive stars, including basic issues such as their formation (Zinnecker \& Yorke, 2007) and end-points (Smith et al. 2010).

We can use observations of populations of massive stars to improve our understanding of the physics and evolution of the stars themselves and, once we are confident of a decent grasp of their behaviour, we can use them as tracers of the properties of their host galaxies. In the following sections, I summarise recent developments in terms of the role of environment on the evolution of massive stars (Sec. 2), observational studies of luminous supergiants in external galaxies (Sec. 3), and the potential of the next generation of ground-based telescopes in studies of massive stars (Sec. 4).

\section{Massive Stars \& Metallicity}

The Large and Small Magellanic Clouds (LMC and SMC) are metal deficient when compared to the solar neighbourhood, with metallicities $(Z)$ of approximately $50 \%$ and 20-25\% solar (e.g. Trundle et al. 2007). This presents us with the chance to study the properties of massive stars in regimes which span a range in metallicity comparable to those found in galaxies in the early universe (e.g. Erb et al. 2006; Nesvadba et al. 2008).

In the course of investigating near-IR photometry from the Spitzer Space Telescope, Bonanos et al. (2009, 2010, these proceedings) have compiled catalogues of published spectral classifications for over 7,000 massive stars in the Clouds. This illustrates the significant progress we have made in determining the stellar content of two of our nearest neighbours. Over the past decade the focus has been to exploit the latest generation of 


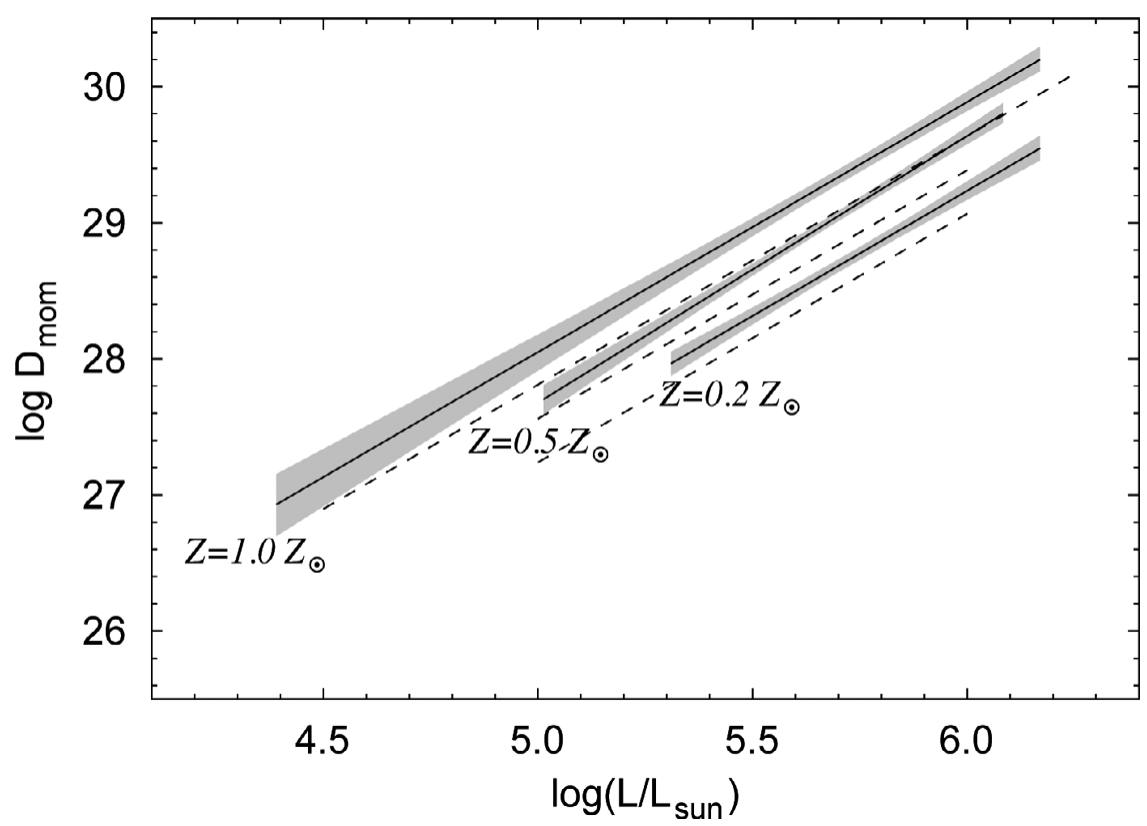

Figure 2. The observed wind-momentum-luminosity relations (solid lines) for O-type stars from the FLAMES survey (Mokiem et al. 2007b) compared with theoretical predictions (dashed lines). The upper, middle and lower relations are the Galactic, LMC and SMC results, respectively.

ground-based telescopes to deliver sufficient spectral resolution and signal-to-noise for quantitative atmospheric analysis of large samples of massive stars in the Clouds. This includes the VLT-FLAMES Survey of Massive Stars (Evans et al. 2005, 2006), and more targeted studies of O-type stars (Massey et al. 2004, 2005, 2009) and B-/Be-type stars (Martayan et al. 2006, 2007). Analysis of these samples has been used to investigate a broad range of parameters, including the $Z$-dependence of stellar wind intensities, effective temperatures, and rotational velocities, each of which is now briefly discussed.

\subsection{Metallicity-dependent stellar winds}

A consequence of the theory of radiatively-driven stellar winds is that their intensities should be dependent on $Z$, with weaker winds at lower metallicities (Kudritzki et al. 1987; Vink et al. 2001). Indications for such an offset were seen from analysis of 22 stars in the Clouds (Massey et al. 2005), with more comprehensive evidence provided by the larger sample from the FLAMES survey (Mokiem et al. 2007b). The FLAMES results are shown in Fig. 2, with fits to the stellar wind-momenta, $\mathrm{D}_{\text {mom }}$ (which is a function of the mass-loss rate, terminal velocity and stellar radius). Fig. 2 also shows the theoretical predictions using the prescription from Vink et al. (2001) - the relative separations of the observed fits are in good agreement, finding a $Z$-dependence with exponents in the range 0.72-0.83 (depending on assumptions regarding clumping in the winds), as compared to $Z^{0.69 \pm 0.10}$ from theory. Analysis of early B-type supergiants in the SMC (Trundle et al. 2004; Trundle \& Lennon 2005) also reveals weaker winds compared to their Galactic counterparts (Crowther, Lennon \& Walborn, 2006).

\subsection{Stellar Effective Temperatures}

The effects of line-blanketing are reduced at lower metallicities due to the diminished cumulative opacity from the metal lines. This leads to less 'back warming' by trapped radiation, thus requiring a hotter model to reproduce the observed line ratios. 
The consequences of this are clearly seen in the temperatures obtained for O-type stars in the SMC, which are hotter than those found for Galactic stars with the same spectral type (Massey et al. 2005; Mokiem et al. 2006). The temperatures for stars in the LMC are seen to fall neatly between the SMC and Galactic results (Mokiem et al. 2007a). A similar $Z$-dependence was also found in the effective temperatures derived for B-type stars observed with FLAMES (Trundle et al. 2007). Typical differences from this effect are $5-10 \%$ when moving from solar to SMC metallicity. Note that there are also temperature differences for cooler supergiants (e.g. Evans \& Howarth, 2003; Levesque et al. 2006), but in the opposite sense and for different reasons (see Evans, 2009).

\subsection{Stellar rotational velocities}

Stellar rotation strongly affects the evolution of all O- and B-type stars via, for example, changes in their main-sequence lifetimes (Meynet \& Maeder, 2000). The effects of rotation also manifest themselves via mixing of chemically-processed material, leading to changes in the surface abundances of elements such as nitrogen (e.g. Brott et al., these proceedings).

The increased fraction of Be- to normal B-type stars at lower metallicity (Maeder, Grebel \& Mermilliod, 1999) pointed to a $Z$-dependence in stellar rotation rates. Investigations into the effects of $Z$ on stellar rotational rates have largely focussed on studies of B-type stars, to avoid the potential complications of angular momentum loss due to strong stellar winds in more massive stars. In the Magellanic Clouds it can be difficult to define an appropriate 'field' or 'cluster' sample for comparison, given that the rotation rates for stars in clusters appear faster than for those in the field population (Keller, 2004; Strom et al. 2005; Wolff et al. 2008). However, a trend of faster velocities at lower $Z$ appears to be borne out by comparisons between rotation rates in the Galaxy and the LMC (Keller, 2004) and, more recently, including the SMC (Martayan et al. 2007; Hunter et al. 2008).

Mokiem et al. (2006) found tentative evidence for different rotational velocity distributions for O-type stars in the SMC compared to the Galaxy, but with some reservations given the potential effects of mass-loss and macroturbulence. New results suggest that macroturbulence is ubiquitous in O-type stars (e.g. Simón-Díaz et al., these proceedings), with Penny \& Gies (2009) suggesting that its magnitude is also $Z$-dependent. This perhaps points to an origin similar to the convective effects argued by Cantiello et al. (2009) to account for microturbulence in massive stars (also seen to have a $Z$-dependence).

\subsection{What next?}

The VLT-FLAMES Tarantula Survey is a new ESO Large Programme which has obtained multi-epoch spectroscopy of over 1,000 stars in the 30 Doradus region of the LMC (Evans et al. 2010a). 30 Dor is the largest H II region in the Local Group, providing us with an excellent stellar nursery to build-up a large observational sample of the most massive stars; the top-level motivations for the survey are summarised by Lennon et al. (these proceedings).

Multi-epoch, radial velocity studies of OB-type stars in Galactic clusters have found binary fractions in excess of $50 \%$ (see Sana \& Evans, these proceedings), and the results of Bosch, Terlevich \& Terlevich (2009) suggest a similarly large binary fraction for the O-type stars in 30 Dor. The Tarantula Survey was designed to combine high-quality spectroscopy for quantitative atmospheric analysis, with repeat observations for detection of massive binaries (over a longest baseline of one year).

This strategy has already paid dividends in terms of putting strong constraints on the nature of 30 Dor \#016, a massive O2-type star on the western fringes of 30 Dor 
Table 1. Summary of published spectroscopic observations and analyses of luminous blue supergiants in external galaxies from the Araucaria project.

\begin{tabular}{|c|c|c|c|}
\hline Galaxy & $\mathrm{d}[\mathrm{Mpc}]$ & $12+\log (\mathrm{O} / \mathrm{H})$ & References \\
\hline IC 1613 & 0.7 & $7.90 \pm 0.08$ & Bresolin et al. (2007) \\
\hline WLM & 0.9 & $7.83 \pm 0.12$ & Bresolin et al. (2006), Urbaneja et al. (2008) \\
\hline NGC 3109 & 1.3 & $7.76 \pm 0.07$ & Evans et al. (2007) \\
\hline NGC 300 & 1.9 & - & Bresolin et al. (2002); Urbaneja et al. (2003); Urbaneja et al. (2005a) \\
\hline NGC 55 & 1.9 & - & Castro et al. (2008) \\
\hline
\end{tabular}

(Evans et al. 2010b). Previous spectroscopy revealed a peculiar radial velocity, but the new FLAMES spectra enabled a massive companion to be ruled out to a high level of confidence, suggesting the star as an ejected runaway.

A further example from the new survey is the observation of two separate components in some of the spectra of R139 (Taylor et al., in preparation). R139 is just over $1^{\prime}$ to the north of R136, the dense cluster at the core of 30 Dor, and was classified by Walborn \& Blades (1997) as O7 Iafp. Previously reported as a single-lined binary with a period of $52.7 \mathrm{~d}$ (Moffat, 1989), recent efforts by Schnurr et al. (2008) found no evidence for binarity in R139 (within a range of periods of up to $200 \mathrm{~d}$ ), although they noted it as having a slightly variable radial velocity. Chené et al. (these proceedings) also report new observations of radial velocity variations in R139 from a separate monitoring campaign.

\section{Beyond the Magellanic Clouds}

The BA-type supergiants are the most intrinsically luminous ('normal') stars at optical wavelengths. The $8-10 \mathrm{~m}$ class telescopes have given us the means by which we can obtain spectroscopy for quantitative analysis of individual blue supergiants in galaxies well beyond the Magellanic clouds, providing us with estimates of their physical parameters, chemical abundances, and also providing an alternative distance diagnostic in the form of the flux-weighted gravity-luminosity relationship (Kudritzki, Bresolin \& Przybilla, 2003; Kudritzki et al. 2008).

Analysis of high-resolution spectroscopy of luminous A-type supergiants in each of NGC 6822 (Venn et al. 2001), M31 (McCarthy et al. 1997, Venn et al. 2000), WLM (Venn et al. 2003) and Sextans A (Kaufer et al. 2004) provided some of the first examples of this type of work in external galaxies, with analysis of larger samples of B-type supergiants in M31 by Trundle et al. (2002) and in M33 by Urbaneja et al. (2005b)

More recently, the Araucaria project has combined optical and near-IR imaging of Cepheids with spectroscopy of luminous blue supergiants to refine the distance determinations to nearby galaxies (Gieren et al. 2005). The Araucaria project has obtained low resolution $(\sim 5 \AA)$ optical spectroscopy with the VLT of BA-type supergiants in a number of external galaxies, as summarised in Table 1.

This has provided us with estimates of oxygen abundances/metallicities in the irregular dwarf galaxies IC 1613 and WLM, and in the main body of NGC 3109. In the larger spirals, the stellar abundances allow study of the radial abundance trends (e.g. Urbaneja et al. 2005a), providing a useful comparison for nebular abundance determinations. In the case of NGC 300, Bresolin et al. (2009) find that 'strong-line' nebular diagnostics (such as the $R_{23}$ ratio) overestimate the true abundance by a factor of two (or more) compared to estimates from auroral nebular lines and the stellar analyses, highlighting the caution that should be employed in metallicity estimates in distant star-forming galaxies (e.g. Kewley \& Ellison, 2008). 


\section{Future Prospects in the Era of ELTs}

Observations from the Araucaria project highlight the power of using individual stars to investigate the properties of their host galaxies. Indeed, one of the most remarkable observations in this context is the VLT spectroscopy of two luminous supergiants in NGC 3621, at a distance of $6.7 \mathrm{Mpc}$ (Bresolin et al. 2001). However, with the exception of the very brightest stars, our potential of exploring the massive star content of external galaxies is limited by the sensitivity of current facilities. With primary apertures in excess of $20 \mathrm{~m}$, the ELTs, the next generation of optical-IR telescopes, will revolutionise our ground-based capabilities, particularly when coupled with adaptive optics (AO) to correct for atmospheric turbulence, thus delivering huge gains in both sensitivity and angular resolution.

Spectroscopy of stellar populations in external galaxies is one of the key elements of the science cases toward the ELTs. This includes using stars as tracers of properties of the host galaxies, and to extend our studies of environmental effects on stellar evolution in systems such as starbursts and very metal-poor galaxies. Efforts toward building the ELTs are increasingly global, with three projects now in the advanced stages of their design, fundraising and planning for instrumentation - the Giant Magellan Telescope (GMT), the Thirty Meter Telescope (TMT), and the European Extremely Large Telescope (E-ELT).

\subsection{MAD: An AO pathfinder for ELTs}

An integral part of the ELTs is the use of AO to deliver improved image quality in the near-IR, and there is an understandable desire to maximise the field-of-view over which one can obtain both good and uniform correction. A key technical development in plans toward the E-ELT was an on-sky demonstration of multi-conjugate adaptive optics (MCAO), which uses multiple deformable mirrors to correct for different layers of turbulence in the atmosphere. This was realised as the Multi-conjugate Adaptive optics Demonstrator (Marchetti et al. 2007), delivering AO-corrected near-IR imaging over a $1 \times 1$ arcmin field. MAD was commissioned at the VLT in early 2007 .

As part of a science demonstration programme, MAD was used to obtain $H$ - and $K_{\mathrm{s}}$ band imaging of R136 (Campbell et al. 2010). The exquisite resolution achieved in these data is shown by Fig. 3, in which the two central WN5h stars in the core of R136 ('a1' and 'a2', separated by 0 '. 1 ) are spatially resolved. This provides near-comparable angular resolution to optical imaging with the $H S T$ (e.g. Hunter et al. 1995), at wavelengths less affected by the significant and variable extinction toward 30 Dor.

When combined with AO-corrected IFU spectroscopy from Schnurr et al. (2009), these data have been used by Crowther et al. (2010) to argue that three of the central stars in R136 have current masses in excess of $150 \mathrm{M}_{\odot}$. From cluster IMF simulations they find the massive stars in R136 are consistent with an upper initial mass-limit of $\sim 300 \mathrm{M}_{\odot}$. This contrasts with the claim by Figer (2005) of an upper limit of $150 \mathrm{M}_{\odot}$ from analysis of the Arches cluster. To address this difference, Crowther et al. returned to the most massive members of the Arches with new photometry and revised distance/extinction estimates, finding greater current masses than those from Martins et al. (2008), and consistent with evolutionary tracks for stars with initial masses in excess of $150 \mathrm{M}_{\odot}$.

\section{Summary}

Work continues apace in characterising the massive star populations in external galaxies (e.g. Herrero et al. 2010) and to improve our understanding of stellar evolution in massive O-type stars (e.g. the Tarantula Survey). We now have a handle on the role of metallicity on some key aspects of stellar evolution, but there remain a number of 


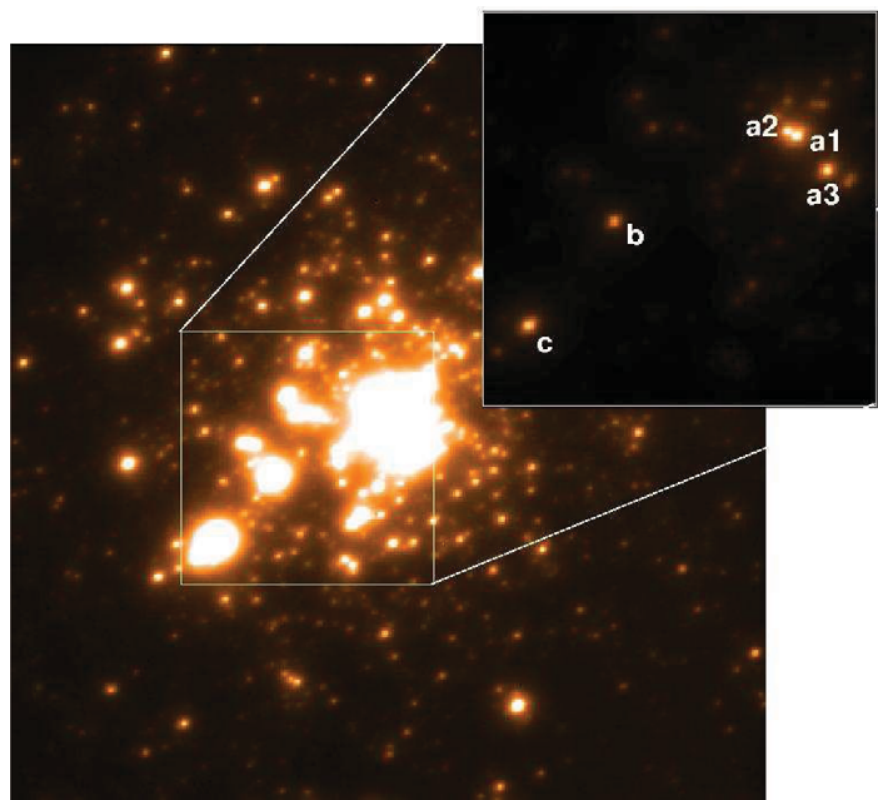

Figure 3. Central $12^{\prime \prime} \times 12^{\prime \prime}$ (eqv. $3 \times 3 \mathrm{pc}$ ) of the $K_{\mathrm{s}}$-band MAD image, with an expanded view of the central $4^{\prime \prime} \times 4^{\prime \prime}$ showing the four WN5h very massive stars analysed by Crowther et al. (2010; components a1, a2, a3, and c).

issues to be addressed before we can claim to really understand 'populations' in distant galaxies, such as the effects of binary evolution and revision to the accepted upper mass limit for single stars.

Inclusion for the effects of binary evolution has been argued to account for some of the observed properties of low-redshift galaxies (e.g. Han, Podsiadlowski \& Lynas-Gray, 2007; Brinchmann, Kunth \& Durret, 2008). The high incidence of massive binaries (Sana \& Evans, these proceedings) suggests further work is required to characterise their typical properties and assess their impact on our interpretation of unresolved populations.

Lastly, the new results of Crowther et al. (2010) urge for investigation of the effects of very massive stars in population synthesis codes. Although very rare, and almost certainly short-lived, Crowther et al. show that the four WN5h stars at the core account for $\sim 45 \%$ of the Lyman-continuum ioinizing flux and $\sim 35 \%$ of the mechanical power of the respective totals from stars in the central $5 \mathrm{pc}$ of R136. The inferred star-formation rates for the components of high-redshift galaxies from Swinbank et al. (2010) and Jones et al. (2010) are an order of magnitude larger than that from 30 Dor but one could, very naively, image scaling-up the density and intensity of R136 to larger scales - in which case the initial mass function would suggest a considerable input of radiation/energy from very massive stars.

\section{Acknowledgements}

I gratefully acknowledge financial support from the IAU.

\section{References}

Bonanos, A. Z., Massa, D. L., Sewilo, M., Lennon, D. J. et al. 2009, AJ, 138, 1003

Bonanos, A. Z., Lennon, D. J., Köhlinger, F., van Loon, J. T. et al. 2010, AJ, 140, 416

Bosch, G., Terlevich, E., \& Terlevich, R. 2009, AJ, 137, 3437 
Bresolin, F., Kudritzki, R.-P., Mendez, R. H., \& Przybilla, N. 2001, ApJ (Letters), 548, L159 Bresolin, F., Gieren, W., Kudritzki, R.-P., Pietrzyński, G. et al. 2002, ApJ, 567, 277 Bresolin, F., Pietrzyński, G., Urbaneja, M. A., Gieren, W. et al. 2006, ApJ, 648, 1007 Bresolin, F., Gieren, W., Kudritzki, R.-P., Pietrzyński, G. et al. 2009, ApJ, 700, 309 Bresolin, F., Urbaneja, M. A., Gieren, W., Pietrzyński, G. et al. 2007, ApJ, 671, 2028 Brinchmann, J., Kunth, D., \& Durret, F. 2008, A\& A, 485, 657

Campbell, M. A., Evans, C. J., Mackey, A. D., Gieles, M. et al. 2010, MNRAS, 405, 421

Cantiello, M., Langer, N., Brott, I., de Koter, A. et al. 2009, A\&A, 499, 279

Castro, N., Herrero, A., Garcia, M., Trundle, C. et al. 2008, A\& A, 485, 41

Crowther, P. A., Lennon, D. J. \& Walborn, N. R. 2006, A\& A, 446, 279

Crowther, P. A., Schnurr, O., Hirschi, R., Yusof, N. et al. 2010, MNRAS 408, 731

Erb, D. K., Shapley, A. E., Pettini, M., Steidel, C. C. et al. 2006, ApJ, 644, 813

Evans, C. J. \& Howarth, I. D. 2003, MNRAS, 345, 1223

Evans, C. J., Smartt, S. J., Lee, J.-K., Lennon, D. J. et al. 2005, A $\& A, 437,467$

Evans, C. J., Lennon, D. J., Smartt, S. J. \& Trundle, C. 2006, A\&A, 456, 623

Evans, C. J., Bresolin, F., Urbaneja, M. A., Pietrzyński, G. et al. 2007, ApJ, 659, 1198

Evans, C. J. 2009, in: J. T. van Loon \& J. M. Oliveira (eds.), The Magellanic System: Stars, Gas, and Galaxies, IAU Symposium 256, p. 325

Evans, C. J., Bastian, N., Beletsky, Y., Brott, I. et al. 2010a, in: R. de Grijs \& J. R. D. Lépine (eds.), Star clusters: basic galactic building blocks throughout time and space, IAU Symposium 266 , p. 35

Evans, C. J., Walborn, N. R., Crowther, P. A., Hénault-Brunet, V. et al. 2010b, ApJ (Letters), $715, \mathrm{~L} 74$

Figer, D. F. 2005, Nature, 434, 192

Gieren, W., Pietrzynski, G., Bresolin, F., Kudritzki, R.-P. et al. 2005, The Messenger, 121, 23

Han, Z., Podsiadlowski, P. \& Lynas-Gray, A. E. 2007, MNRAS, 380, 1098

Herrero, A., Garcia, M., Uytterhoeven, K., Najarro, F. et al. 2010, A\&A, 513, A70

Hunter, D. A., Shaya, E. J., Holtzman, J. A., Light, R. M. et al. 1995, ApJ, 448, 179

Hunter, I., Lennon, D. J., Dufton, P. L., Trundle, C. et al. 2008, A\&A, 479, 541

Jones, T. A., Swinbank, A. M., Ellis, R. S., Richard, J. et al. 2010, MNRAS, 404, 1247

Kaufer, A., Venn, K. A., Tolstoy, E., Pinte, C. et al. 2004, AJ, 127, 2723

Keller, S. C. 2004, PASA, 21, 310

Kewley, L. J. \& Ellison, S. L. 2008, ApJ, 681, 1183

Kudritzki, R. P., Pauldrach, A. \& Puls, J. 1987, A\&A, 173, 293

Kudritzki, R. P., Bresolin, F. \& Przybilla, N. 2003, ApJ (Letters), 582, L83

Kudritzki, R.-P., Urbaneja, M. A., Bresolin, F., Przybilla, N. et al. 2008, ApJ, 681, 269

Leitherer, C., Schaerer, D., Goldader, J. D., González Delgado, R. M. et al. 1999, ApJS, 123, 3

Leitherer, C., Ortiz Otálvaro, P. A., Bresolin, F., Kudritzki, R.-P. et al. 2010, ApJS, 189, 309

Levesque, E. M., Massey, P., Olsen, K. A. G., Plez, B. et al. 2006, ApJ, 645, 1102

Maeder, A., Grebel, E. K. \& Mermilliod, J.-C. 1999, A\&A, 346, 459

Marchetti, E., Brast, R., Delabre, B., Donaldson, R. et al. 2007, The Messenger, 129, 8

Martayan, C., Frémat, Y., Hubert, A.-M., Floquet, M. et al. 2006, A\&SA, 452, 273

Martayan, C., Frémat, Y., Hubert, A.-M., Floquet, M. et al. 2007, A\&SA, 462, 683

Martins, F., Hillier, D. J., Paumard, T., Eisenhauer, F. et al. 2008, A\&BA, 478, 219

Massey, P., Zangari, A. M., Morrell, N. I., Puls, J. et al. 2009, ApJ, 692, 618

Massey, P., Puls, J., Pauldrach, A. W. A., Bresolin, F. et al. 2005, ApJ, 627, 477

Massey, P., Bresolin, F., Kudritzki, R. P., Puls, J. et al. 2004, ApJ, 608, 1001

McCarthy, J. K., Kudritzki, R.-P., Lennon, D. J., Venn, K. A. et al. 1997, ApJ, 482, 757

Meynet, G. \& Maeder, A. 2000, A\&A, 361, 101

Moffat, A. F. J. 1989, ApJ, 347, 373

Mokiem, M. R., de Koter, A., Evans, C. J., Puls, J. et al. 2006, A\&AA, 456, 1131

Mokiem, M. R., de Koter, A., Evans, C. J., Puls, J. et al. 2007a, A $\& A$, 465, 1003

Mokiem, M. R., de Koter, A., Vink, J. S., Puls, J. et al. 2007b, A\& $A, 473,603$

Nesvadba, N. P. H., Lehnert, M. D., Davies, R. I., Verma, A. et al. 2008, A\&A A, 479, 67 
Pauldrach, A. W. A., Hoffmann, T. L. \& Lennon, M. 2001, A\& A, 375, 161

Penny, L. R. \& Gies, D. R. 2009, ApJ, 700, 844

Pettini, M., Rix, S. A., Steidel, C. C., Adelberger, K. L. et al. 2002, ApJ, 569, 742

Schnurr, O., Moffat, A. F. J., St-Louis, N., Morrell, N. I. et al. 2008, MNRAS, 389, 806

Schnurr, O., Chené, A.-N., Casoli, J., Moffat, A. F. J. et al. 2009, MNRAS, 397, 2049

Smith, N., Li, W., Filippenko, A. V. \& Chornock, R. 2010, ArXiv e-prints 1006, .

Strom, S. E., Wolff, S. C. \& Dror, D. H. A. 2005, AJ, 129, 809

Swinbank, A. M., Smail, I., Longmore, S., Harris, A. I. et al. 2010, Nature, 464, 733

Trundle, C., Dufton, P. L., Lennon, D. J., Smartt, S. J. et al. 2002, A\&GA, 395, 519

Trundle, C., Lennon, D. J., Puls, J., \& Dufton, P. L. 2004, A\&A, 417, 217

Trundle, C. \& Lennon, D. J. 2005, A\& $A$, 434, 677

Trundle, C., Dufton, P. L., Hunter, I., Evans, C. J. et al. 2007, A\&SA, 471, 625

Urbaneja, M. A., Herrero, A., Bresolin, F., Kudritzki, R.-P. et al. 2003, ApJ (Letters), 584, L73

Urbaneja, M. A., Herrero, A., Bresolin, F., Kudritzki, R.-P. et al. 2005a, ApJ, 622, 862

Urbaneja, M. A., Herrero, A., Kudritzki, R.-P., Najarro, F. et al. 2005b, ApJ, 635, 311

Urbaneja, M. A., Kudritzki, R.-P., Bresolin, F., Przybilla, N. et al. 2008, ApJ, 684, 118

Venn, K. A., McCarthy, J. K., Lennon, D. J., Przybilla, N. et al. 2000, ApJ, 541, 610

Venn, K. A., Lennon, D. J., Kaufer, A., McCarthy, J. K. et al. 2001, ApJ, 547, 765

Venn, K. A., Tolstoy, E., Kaufer, A., Skillman, E. D. et al. 2003, AJ, 126, 1326

Vink, J. S., de Koter, A., \& Lamers, H. J. G. L. M. 2001, A\&A, 369, 574

Walborn, N. R. \& Blades, J. C. 1997, ApJS, 112, 457

Wolff, S. C., Strom, S. E., Cunha, K., Daflon, S. et al. 2008, AJ, 136, 1049

Zinnecker, H. \& Yorke, H. W. 2007, ARAA, 45, 481 